\title{
Material anchors for conceptual blends
}

\author{
Edwin Hutchins \\ Department of Cognitive Science, University of California, San Diego, \\ 9500 Gilman Drive, La Jolla, CA 92093-0515, USA
}

Received 1 March 2003; received in revised form 19 September 2003; accepted 28 June 2004

\begin{abstract}
Reasoning processes require stable representations of constraints. There are two principal ways to achieve stability in conceptual models. First, the conceptual models that anthropologists call cultural models achieve representational stability via a combination of intrapersonal and interpersonal processes. Second, the association of conceptual structure with material structure can stabilize conceptual representations. This is an old and pervasive cognitive strategy. Conceptual blending theory provides a useful framework for considering the joint contributions and mutual constraints of mental and material structure. Projecting material structure into a blended space can stabilize the conceptual blend. I call an input space from which material structure is projected into a blend a 'material anchor' for the blend. The term material anchor is meant to emphasize the stabilizing role of the material structure. In this article, I will present and discuss a number of examples of materially anchored blends, which depend to different degrees on material structure. Materially anchored blends vary on a number of complexly related dimensions, including the extent to which the blend relies on the presence of material structure in the perceptual field, the complexity of the material structure, and whether the material structure was designed to support the blend or is used opportunistically.
\end{abstract}

(C) 2005 Elsevier B.V. All rights reserved.

Keywords: Blending; Cognitive artifacts; Distributed cognition; Integration; Material anchors

\section{Introduction}

In this article I discuss a general and ancient human cognitive phenomenon, the association of conceptual structure with material structure. Examples range from the linguistic construction of fictive motion, to the cultural construction of queuing or standing

E-mail address: hutchins@gogsci.ucsd.edu. 
in line; from children's games to essential tools of life-or-death endeavors; from the conceptualization of simple everyday logical distinctions to the tools of science and mathematics. All of these seemingly disparate phenomena are instances of a particular way of associating conceptual structure with material structure.

The ability to combine conceptual structure with material structure is a key cognitive strategy. The basic abilities are very old. In fact, non-human primates may be capable of some forms of conceptual and material combination. What are the processes that can do this? What varieties of combining are possible? What are the cognitive consequences of making such associations?

I have found the framework of conceptual blending (Fauconnier and Turner, 2002) to be especially useful for thinking about the relations between conceptual and material structure. It allows us to take a unified view of phenomena that would have otherwise been considered quite disparate and frames the relations among conceptual and material structures in ways that make it possible to answer some of the questions posed above.

A few words about what this approach is not. Material structure can have many kinds of relations with conceptual structure. One of the most common is the relationship of representation. All of language depends on this sort of relationship so this one comes easily to mind for linguists. Spoken or written structure in the world represents conceptual entities. This immediately opens the door to the field of semiotics and the huge literature on representations. But before jumping directly to signs and symbols it is useful to examine some more basic processes by which meaning is attached to or projected onto material structure. The relation we are looking for here is not quite the same as the 'representation' function described in the preceding phrase. I will return to this topic at the end of the article.

In order to see this other sort of relation, it is necessary to introduce the basics of conceptual blending. I will leave it to other authors in this volume to present the details of that theory.

Fauconnier (1997) describes the essence of the process as follows:

A conceptual blend operates in two input mental spaces to yield a third space, the blend. Partial structure from the input spaces is projected into the blended space, which has emergent structure of its own. The emergent structure arises in three interrelated ways:

Composition: Taken together, the projections from the inputs make new relations available that did not exist in the separate inputs. Completion: Knowledge of background frames, cognitive and cultural models, allows the composite structure projected into the blend from the inputs to be viewed as part of a larger self-contained structure in the blend. The pattern in the blend triggered by the inherited structures is 'completed' into the larger, emergent structure. Elaboration: The structure in the blend can then be elaborated. This is called 'running the blend.' It consists of cognitive work performed within the blend, according to its own emergent logic. (Fauconnier, 1997: 150-151)

The cases collected in this article show how material structure provides an input space that is blended with other inputs. The principles of composition, completion, and elaboration will figure prominently in these examples. Conceptual blending theory makes 

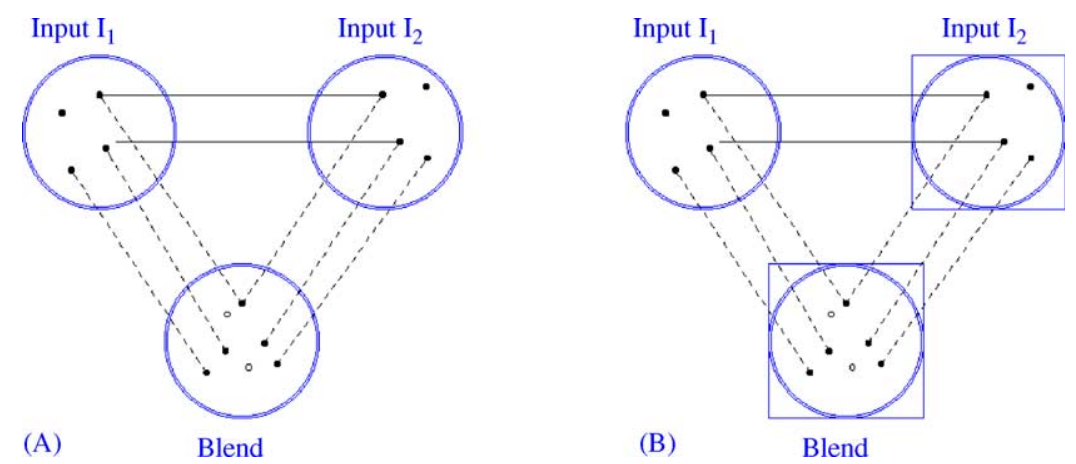

Fig. 1. A conventional conceptual blend (A), and a conceptual blend with a material anchor (B).

it clear that the material input space need not be a representation of the concepts in the input space with which it is blended. Fig. 1 illustrates these relations in schematic form. Fig. 1A is a depiction of a conventional conceptual blend with two conceptual input spaces and a blended space. Fig. 1B introduces a new notation. ${ }^{1}$ The square around input space 2 indicates that material structure is contributed by that input space to the blended space.

\section{The problem of conceptual stability}

Thinking processes sometimes involve complex manipulations of conceptual structure. Conceptual structure must be represented in a way that allows some parts of the representation to be manipulated, while other parts remain stable. The complexity of the manipulations of structure can be increased if the stability of the representations can be increased. The stability of the representations is a necessary feature of the reasoning process, but it is often taken for granted. The need for representational stability becomes more visible in circumstances where the necessary stability is not present.

\section{Culturally based reasoning}

In the 1960s and 1970s a movement arose in cognitive psychology investigating the biases and limitations of human reasoning processes (see Kahneman et al., 1982). For example, the famous four-card task, originally developed by Wason (1966), demonstrated that otherwise intelligent subjects (university undergraduates) had surprising amount of difficulty reasoning about logical relations. This study triggered a large number of followup studies of reasoning and inference. Of these, one clearly showed the role of a cultural heritage of mental models in the abilities of individuals to reason correctly. D'Andrade (1989) considered a number of logical isomorphs consisting of a premise in the form of an

\footnotetext{
${ }^{1}$ This notation was first used by Robert F. Williams in his thesis proposal to the Department of Cognitive Science, University of California, San Diego, in June, 2003.
} 
'if-then' statement followed by an observation and a set of potential conclusions. For example, a subject is shown a premise, "If $\mathrm{x}$ is true then $\mathrm{y}$ is true" and then an observation, "We know that $\mathrm{y}$ is not true". The subject is then asked what can be inferred about $\mathrm{x}$ from the observation that $\mathrm{y}$ is not true. The choices are: " $\therefore$ It is the case that $\mathrm{x}$ is true." (invalid conclusion), " $\because$ We cannot know if $\mathrm{x}$ is true or not." (invalid conclusion), and " $\because$ It is the case that $\mathrm{x}$ is not true." (valid conclusion). Only a small minority (about 15\%) of subjects choose the correct conclusion.

This example illustrates an abstract premise. From D'Andrade's perspective, what is important about the premise in this problem is that, for most subjects, it is not culturally coherent. That is, unless $\mathrm{x}$ and $\mathrm{y}$ are associated with particular known concepts, our culture has nothing in particular to say about the relationship between $\mathrm{x}$ and $\mathrm{y}$. Contrast this with a culturally coherent isomorph:

Given the premise, "If this is a garnet, then it is a semi-precious stone" and the observation, "This is not a semi-precious stone", most subjects correctly reject the conclusions " $\because$ This is a garnet." (invalid conclusion) and " $\because$ We can't know if this is a garnet or not." (invalid conclusion), preferring the correct conclusion " $\because$ This is not a garnet." Formally the two problems are identical. A formal reasoning system that can solve one correctly should be able to solve the other correctly.

In a series of experiments, D'Andrade showed that university students can draw correct logical inferences when such problems are expressed in terms of culturally coherent mental schemas. D'Andrade hypothesized that the ability to reason correctly with culturally coherent materials is due to the stability of the representation of the premises. Performing the modus tollens inference (If $\mathrm{x}$ then $\mathrm{y}$; not $\mathrm{x}$, therefore not $\mathrm{y}$ ), as illustrated in the two examples above requires the subject to make two shifts in perspective. First from the ' $x$ ' case to the ' $y$ ' case, and then from the ' $y$ ' case to the 'not y' case. ${ }^{2}$ It appears that premises that lack cultural coherence cannot be held stably in memory while the transformations required to make the inferences are performed.

D'Andrade's study indicates that one way to establish the stability of representation to facilitate reasoning is to express the problems in terms of familiar cultural models. Because they are shared, cultural models tend to be supported and reinforced by the behavior and thinking of others. Cultural models are also systematic in the sense that they exist in a complex nexus of models that mutually constrain one another. Most cultural models are closely related to many other models. This inter-linking contributes to the conservatism of cultural beliefs over time and to the stability of cultural models as resources for individual and group reasoning processes.

\section{Material anchors for conceptual blends}

There is another way to increase the stability of conceptual representations. Cultural models are not only ideas that reside inside minds, they are often also embodied in material

\footnotetext{
${ }^{2}$ Other theories of reasoning posit other operations, but in all cases, stable representation of the relation given in the premise is required and modus tollens inference requires more complex manipulations than modus ponens does.
} 
artifacts. Over the years I have collected a number of examples of thinking strategies that involve the interaction of mental structure and material structure. I only recently realized that the conceptual blending framework provides a means of understanding these examples as members of a larger class of cognitive phenomena. The theory of conceptual blending and mental spaces deals with abstract models of the kinds we usually think of as mental models. The organization of the models supports various inferences. The key thing here is the way in which two or more spaces are blended together. Various elements of the input spaces are selectively mapped to the blended space. In the blended space, new inferences are possible. In fact, some new inferences become automatic. In most treatments of conceptual integration, all input spaces are strictly mental constructs. In this article I would like to explore the possibilities that arise when some or all of the structure contributed by one or more of the input spaces has physical form.

Consider a line of people queuing for theatre tickets. This cultural practice creates a spatial memory for the order of arrival of clients. The participants use their own bodies and the locations of their bodies in space to encode order relations. The gestalt principle of linearity makes the line configuration perceptually salient. Our perceptual systems have a natural bias to find line-like structure. But seeing a line is not sufficient to make a queue. Not all lines are queues. Soldiers standing at attention in formation form a line, but not a queue. In order to see a line as a queue, one must project conceptual structure onto the line. The conceptual structure is the notion of sequential order. For our purposes, we will represent this directional ordering as a trajector (Langacker, 1987). Conceptually blending the physical structure of the line with an imagined directional trajector turns the line into a queue (see Fig. 2).

Let's use this example to reexamine the three ways in which structure emerges in a blend. Composition: the sequential ordering of elements in the queue emerges from the blend of the line with the trajector. This ordering of elements was not present in either input to the blended space. Blending with a linear trajector makes a line into a queue. The queue exists only when the line is blended with the trajector. In the blended space, the line is experienced as an ordered queue. Phenomenologically, the object that emerges from the blending process, that is the queue, is experienced in the perception of the material structure; one sees a queue rather than a line. Completion: Other elements are recruited so that seeing the queue 'makes sense' in the cultural context of people seeking a service and the (far from universal) cultural principle of "first-come, first-served". Elaboration: Once the emergent structure of the queue is developed, other reasoning operations become possible. For example, the questions: Who is first in line? Who is last? How many people are in front of me? can be answered when the line is experienced as a queue, but not when it is simply a line.

This brief example raises some fundamental issues about the relationships among conceptual and perceptual processes, between mental and material structure. From the perspective of the tradition of representationalism in cognitive science it is tempting to imagine that the two inputs to the blend are mental conceptual structure on the one hand and a mental representation of the material structure on the other. This is the approach taken by Liddel (2003) and Taub (2001) in their analyses of ASL signs. For Liddell, real space labels "a person's current conception of the immediate environment based on sensory input." (Liddel, 2003: 82). Similarly, in a discussion of iconicity, Taub says that 


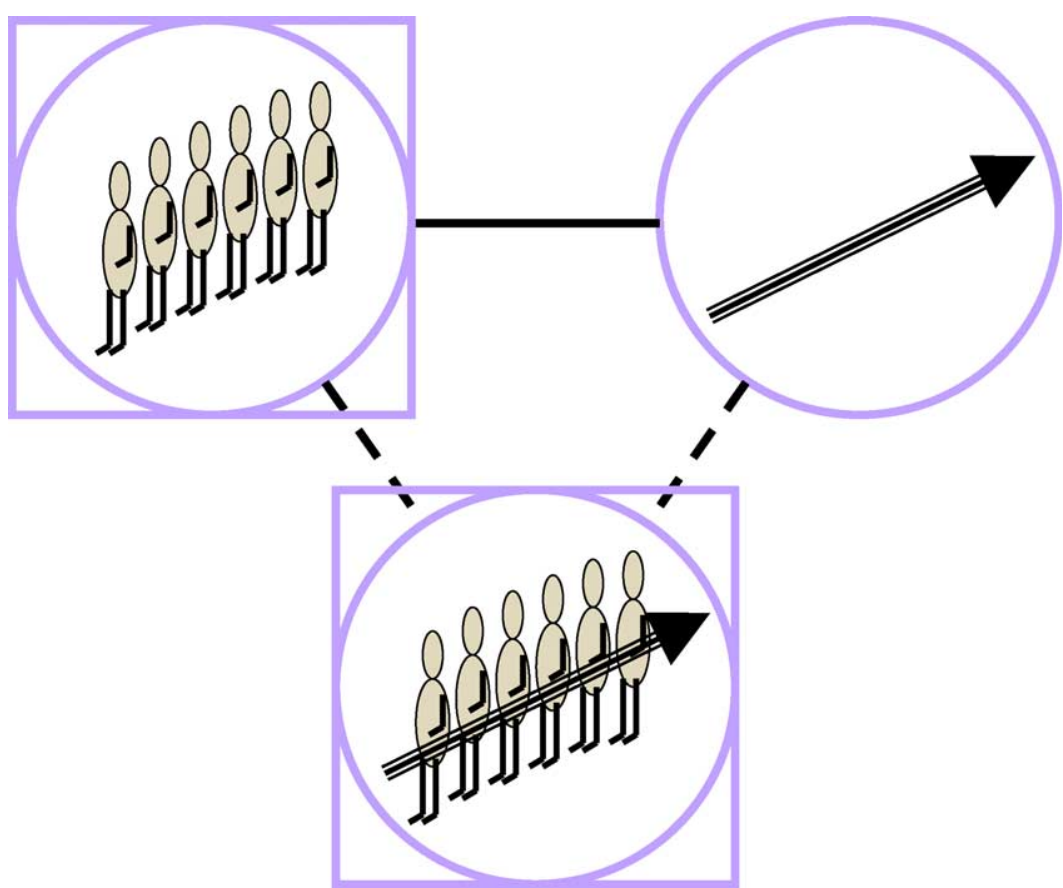

Fig. 2. The conceptual blend of a trajector with a line of people, forming a queue in the blended space.

iconicity is not an objective relation between the form of a sign and its meaning, it is a relation between our conception of a sign and our conception of it's meaning (Taub, 2001: 19). Taub holds iconicity to be a phenomenon of real space. This approach to the formation of conceptual blends that seem to rely on material structure holds that inputs to the blend are (1) a mental representation of the material structure and (2) a mental representation of whatever conceptual elements are present. For Liddell and Taub, real space is the first of these inputs.

However, it is useful to remember Rodney Brooks' (1991) observation that "the world is its own best model." Not only is there no need to posit a separate mental representation of the material structure as an input space, doing so obscures some important phenomena. Another alternative is to say that the physical objects themselves are input to the conceptual blending process. This is what I intend when I speak of 'material anchors' for conceptual blends. What is at stake here is the boundary of the conceptual blending process. Shall the conceptual blending process be an entirely conceptual process that operates on ('real space' as delivered to the process in the form of) the output of perceptual processes, or shall the conceptual blending process include the perceptual processes and therefore include the bodily interaction with the physical world. The blending diagrams implied by the two frameworks are depicted in Fig. 3. Fig. 3A shows the 'real space' interpretation of the role of material structure in conceptual blending. Fig. 3B depicts the relationship suggested by the phrase 'material anchor for a conceptual blend.' 

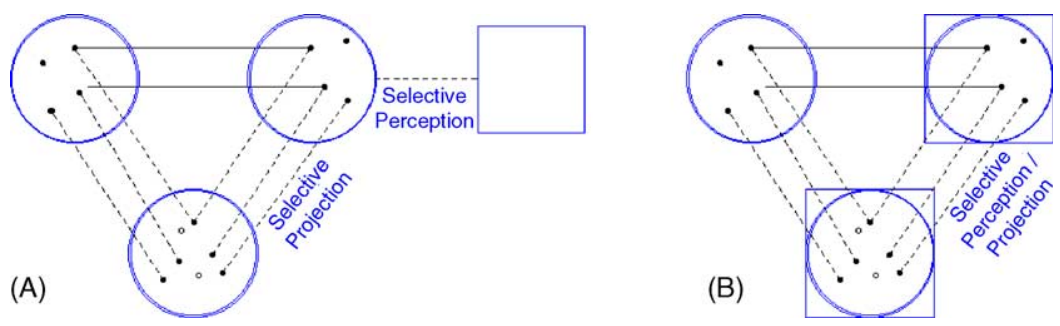

Fig. 3. The 'real space' interpretation of the contribution of material structure to conceptual blending (A), and the 'material anchors' interpretation (B).

What are the advantages of these two different ways of thinking of the role of material structure in the formation of conceptual blends? One of the problems with the 'real space' formulation is that it requires two stages of selectivity. First there is the selectivity of perception that produces a filtered conceptual representation of the physical world. Second, there is selective projection in the process by which the prior conceptualization of the world (the "real space" representation) is blended with the other conceptual input. Is there any evidence that these are two separate processes? It seems preferable to assume that the selective attention to, and projection of, structure from the material world to the blended space is the perceptual process. That is, that selective perception is a conceptual process.

Consider again the line experienced as a queue. In order to experience the line as a queue and to run the queue blend, there is no need for a person to conceptually represent the details of the composition of the line, because the line is present. The present line can be selectively conceptualized as a queue in the running of the blend. This implies that the selectivity of the projection process that brings structure to the blend goes all the way back to the perception of the object itself. Consider the task of counting the number of people in a line. A standard strategy for counting objects in a line is to conceptually blend a trajector with the structure of the line to produce a queue. Objects are treated in order, and the mental act of noting each one is accompanied by a transition in a sequence of number names, "one, two, ..." Where does this counting happen? The "real space" approach would place it in mental representations of the environment. However, it is obvious that it is not necessary to have a mental representation of the entire line of objects in order to count the objects in the line. Attention is allocated to objects in the queue-defined order. Enough of the queue must be represented to maintain the orientation of the imagined trajector and to maintain some sort of marking on the object of current interest possibly in the context of the adjacent objects. Objects far from the current location of counting need only be weakly represented or might not be represented at all. Perhaps the "real space" framing can be saved by acknowledging that during the counting operation, the contents of "real space" must be continually changing during the composition and elaboration of the blend as guided by the coordination of the counting processes with the orientation of the imagined trajector and with the structure of the object.

The queue emerges from a blend in which one input space is an input space in the usual sense, a conceptual domain consisting of conceptual elements and relations (a trajector through virtual locations). The other input is a structured configuration of material elements (persons in a line). As is the case with all blends, cross-space mappings between 
conceptual and material elements link the two spaces and selective projection from the inputs into the blended space give rise to emergent properties. In addition to the emergent property of sequential relations among the persons in the queue, one of the most important emergent properties of this blend is the stabilization of the representation of these conceptual relations. A mental space is blended with a material structure that is sufficiently immutable to hold the conceptual relationships fixed while other operations are performed. The advantage of the line, over standing in some other configuration, is that a very simple shape of motion of a trajector applied to the line can map the spatial structure of the line into temporal structure. This is why a group of people milling about at a cocktail party is a poor material anchor for the queue blend. Blending with material anchors may increase the stability of conceptual structure, enabling more complex reasoning processes than would be possible otherwise. In some cases, the conceptual structures to be represented and manipulated are so complex that they cannot possibly be given stable representation using mental resources alone. In order to produce and manipulate a stable representation of the conceptual elements involved in such computations, the elements must be somehow held (or anchored) in place. The 'holding in place' is accomplished by mapping the conceptual elements onto a relatively stable material structure. This is how a material medium becomes an anchor for a conceptual blend.

A physical structure is not a material anchor because of some intrinsic quality, but because of the way it is used. It might be better to ask under what conditions something becomes a material anchor than to ask whether it is a material anchor. If conceptual elements are mapped onto a material pattern in such a way that the perceived relationships among the material elements are taken as proxies (consciously or unconsciously) for relationships among conceptual elements, then the material pattern is acting as a material anchor. The conceptual relationships that are mapped into material structure range from the minimum case of individuation (this element is distinguishable from all others) to extremely complex systems of relationships (the scales on a sliderule, for example).

\section{Examples}

To illustrate the roles of mental and material structure in thinking I will focus first on a set of examples that involve the coordination of spatial and temporal structure. Some of the examples come from mundane activities while others (the more complex ones) are found in culturally elaborated contexts. I will begin with blends that are mostly mental and gradually work toward blends that involve the use of more material resources.

As cases involving increasing amounts of material structure are considered, I will show how the use of input spaces that contain material structure can create conceptual blends that permit people to do some astonishing thinking while using fairly simple internal cognitive processes. From an anthropological perspective, I believe that the sorts of processes I describe here are absolutely essential to an understanding of the nature of human cognitive accomplishment. The techniques of blending conceptual and material structure change the ratio of cognitive effort to computational accomplishment. Depending on the environment in which they are deployed, they may either produce increased intellectual accomplishment with high effort or steady output with considerably less effort. 


\subsection{Fictive motion}

Fictive motion (Fauconnier, 1997; Langacker, 1987; Talmy, 1995) is a form of conceptual blend. Consider the sentence, "The fence runs all the way down to the river". In this construction, an input space containing a static scene of a fence and a river is blended with an input space that contributes a moving trajector on a path with a reference point. The moving trajector contributes a shape of motion (movement down a hill to the edge of a river) along the relevant dimension of the static object (the length of the fence). Fauconnier (1997: 177) notes,

This is a remarkable mode of expression: It conveys motion and immobility at the same time. Objective immobility is expressed along with perceptual or conceptual motion (the conceptualizer's gaze, mental scanning, or structure projection).

In the blended space, the fence extends down to the river via fictive motion. A set of correspondences between the features of the shape of the fence and the shape of the motion has been established. Fictive motion blends the shape of a movement through space with the spatial shape of an object to produce a temporal sequence of attention to the shape of the object. In the presence of the object, it guides the perceiver's attention. In the absence of the object, it constructs the shape of the imagined object by giving shape to the conceptualizer's mental scan.

\subsection{Method of loci}

The method of loci provides a well-known example of the cognitive use of material structure. In order to remember a long sequence of ideas, one associates the ideas, in order, with a set of landmarks in the physical environment in which the items will have to be remembered. The method of loci sets up a simple trajectory of attention across a set of features or landmarks of the environment. One may establish a flow through the environment that brings attention to the landmarks in a particular order. This is a layered blend.

In Western culture this technique can be traced back to Aristotle and later, the Roman orator, Cicero. It is clearly a much older technique than that. In the Trobriand Islands of Papua New Guinea, long narratives are structured around the local geography. The protagonist of a story typically travels from village to village. For example, in the story of Baroweni (Hutchins, 1987), Barownei's mother travels from the island of Tuma to the village of Osapoula. In the narrative, she stops in a series of villages that, if plotted on a map, describe a rough line from Tuma to Osapoula. Young people learn something about the geography of the area from the stories, and the narrators seem to use a variant of the method of loci to control the order of events in the narrative. The villages are used as landmarks to remember the parts of the story. Harwood (1976) makes the connection to the method of loci explicit in a paper titled "Cicero in the Trobriands".

The initial input spaces are the shape of the motion of a trajector and the set of landmarks in the environment (see Fig. 4). Together these produce a blend that is the sequential flow through the landmarks in the environment. The sequential relations of the landmarks are an emergent property of this blend. This space then becomes an input space 


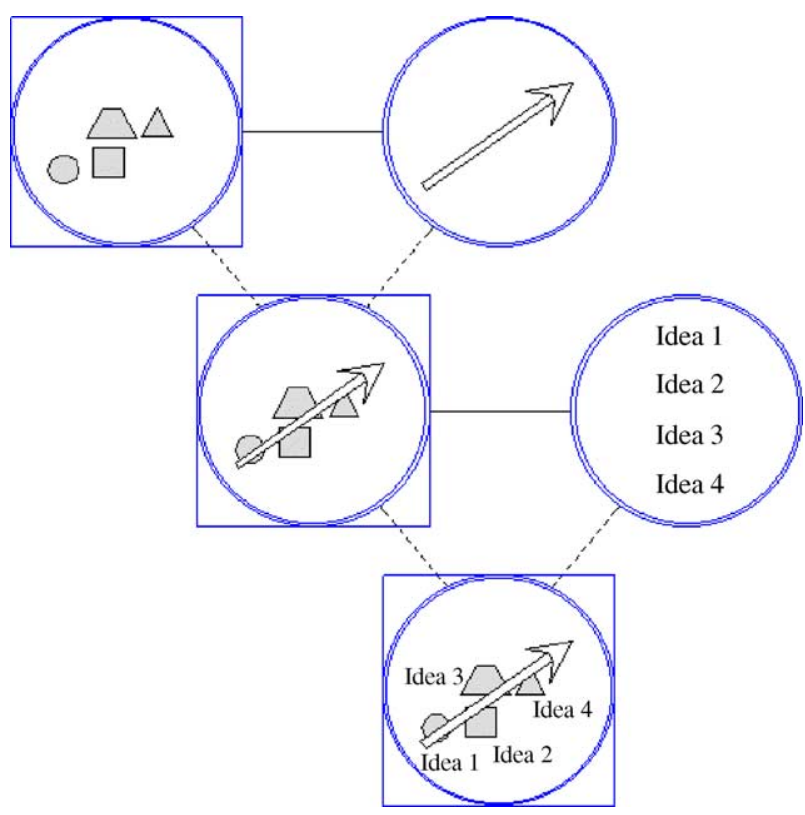

Fig. 4. The compound blend that underlies the use of the method of loci.

for a more complex blend. The items to be remembered are associated with the landmarks producing a space in which the items to be remembered are imagined to be co-located with their corresponding landmarks. In this blended space, the items to be remembered acquire the sequential relations that were created among the landmarks. These sequential relations among the items to be remembered are emergent properties of this compound blended space. Like fictive motion, the method of loci blends the motion of a trajector with objects. In this case, the objects are real rather than imaginary. ${ }^{3}$

The method of loci makes opportunistic use of space. The spatial relations of the landmarks do not contribute any semantic content to the problem. But the landmarks themselves do provide memory cues, and the sequential relations among the landmarks, that were created by mapping a particular shape of motion onto them, is inherited by the set of items to be remembered. As was the case in fictive motion, a blend of a shape of motion with the spatial extent of a set of objects produces temporal ordering among the objects.

Similar strategies are used in many activities where it is important to do a series of actions in order, making sure each action is done once and only once. Pilots inspecting their airplanes before flight follow a simple trajectory around the airplane. The shape of the motion imposes a set of sequential relations on the items to be checked. The cognitive problem that is solved is one of the sequential control of action to produce an action set that is exhaustive and non-repetitive.

\footnotetext{
${ }^{3}$ Fauconnier and Turner (2002) point out that it is also possible to anchor the method of loci with imagined landmarks, as long as the spatial relations among the landmarks are well known.
} 


\subsection{The intelligent use of space}

Fictive motion maps a shape of motion of an imagined trajector onto an imagined object and thereby gives the object shape. In the method of loci, a shape of motion is mapped onto real objects in space to produce sequential relations among those objects. A simple strategy for the disassembly and re-assembly of mechanical devices places real objects in space in such a way that a simple shape of motion will produce a sequential ordering on the objects. Kirsh (1995) describes this technique. During disassembly, as parts are removed from a device, they are placed in order in a line on the assembly space. To reassemble the device, the parts are taken off the line in the reverse direction. This reversal of direction reverses the sequential relations of the parts.

The blending of conceptual structure in the form of an imagined trajector with material arrays of objects to create conceptualized sequences in physical space is an absolutely ubiquitous process. It is also an extremely old cognitive strategy. Wynn (1989) examined stone tools going back several millions of years. He describes an innovation that arose about a million years ago. Tool makers before that could strike a stone repeatedly in nearly the same place. At that time, they began striking a stone a sequence of blows, each one a little further along the stone than the last. This looks like an operational version of the projection of a simple trajector onto the activity of chipping stone tools.

\subsection{Japanese hand calendar}

Nakahara (1996) describes a visualization technique used by Japanese students to compute the day of the week of any day in the year. Exams sometimes contain questions requiring the student to make such a computation. The problem to be solved here concerns the coordination of the weekly cycle of seven day names with the monthly cycles of 28 (29 in the case of a leap year), 30, or 31 days. The computation is accomplished by mapping the cycle of day names and the cycle of month names onto a common structure. A variety of structures might serve this purpose, but the one chosen by Japanese students is a set of regions on the first three fingers of the left hand. The regions are defined by the creases in the fingers that appear at the finger joints. These creases are imagined to make three boxes on each finger. Attending selectively to the first two fingers and the top box on the ring finger creates a field of seven boxes (see Fig. 5). The mapping of month names onto these boxes is invariant. However, the sequence of month names follows a complex trajectory across the seven boxes. It is difficult to visualize this trajector. To make the task of constructing the trajector, and the sequence of month names easier, the mapping is taught as a sequence of motions of the left thumb over the regions of the fingers. This permits the memory for the trajectory to be redundantly stored in visual and in motor memory.

Since the week is seven days long, in any month all of the days that have numbers that are 0 modulo 7 will fall on the same day of the week. For example, in 2002, the 7 th, the 14th, the 21st, and the 28th of August all fall on a Wednesday.

The names of the days of the week can be mapped onto the same structure using the topto-bottom and left-to-right pattern shown. The trajector that constructs the sequence of names of days of the week (top to bottom, left to right) is less complex than the pattern of month names. It is remembered as a visual pattern only. 


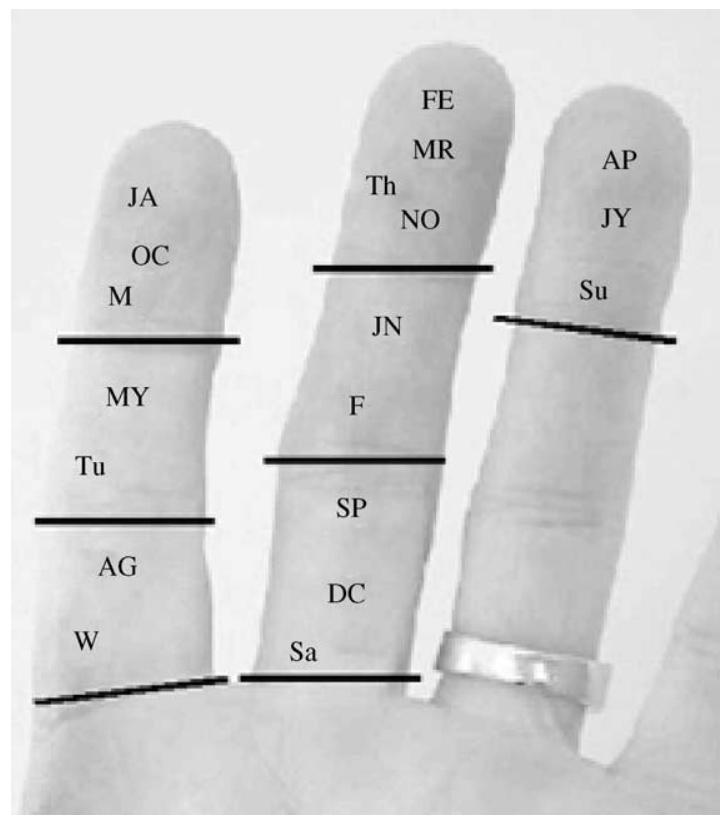

Fig. 5. The mappings of day names and month names onto the fingers of the left hand create a blended space on which the day of the week of any date can easily be computed.

The intersection of this pattern of mapping day names with the pattern for mapping month names produces a complete set of correspondences between day names and month names that captures the effect of the varying lengths of months. Fig. 1 shows the arrangement for 2002. The correspondences of day names and month names change from year to year. To determine the day of the week of an arbitrary date in 2002, say August 23, one simply looks at the hand - while imagining the month and day names - and finds the box containing the month name. The day name in that box is "Wednesday". This means that all of the Wednesdays in August of 2002 fall on dates that are 0 modulo 7 . Thus, the $7 \mathrm{th}$, the $14 \mathrm{th}$, the $21 \mathrm{st}$, and the 28th of August are Wednesdays. Imagine the $21 \mathrm{st}$ of August.

It's a trivial computation to determine that the 21 st is a Wednesday. The 23rd falls two days later, so one can count forward two days, and determine that the 23rd is a Friday.

Mapping the day and month names onto the hand composes the blended space with its emergent properties - the correspondences between day names and months. Locating the landmark date and counting with respect to it are processes that elaborate or 'run' the blend. Remember that Fauconnier (1997: 151) said that running the blend, "consists in cognitive work performed within the blend, according to its own emergent logic." The day of the week of any date in the year can easily be determined by locating a nearby known landmark and counting up or down to the desired date. Dates such as the 10th or the 25th that have values that are 3 or 4 modulo 7 require more counting and more memory resources than dates that are nearer 0 modulo 7 . For cases that require much counting, the 
ability to do the counting on the fingers itself provides a considerable reduction in the memory demands of the task.

This technique makes opportunistic use of the hand as a material anchor for the superimposition of two related structures. The hand itself does not provide any of the semantic content of the task. Processes that blend a trajector with spatial locations are combined with conventional name sequences to map the patterns of month and day names onto the hand. These mappings construct the composite image. Additional conventional cultural procedures (finding '0-modulo-7' landmarks and counting up or down in coordination with shifts in locus of attention) are then applied to the image to compute results in terms of another cultural convention (the day of the week of any date). Without the hand, it would be difficult to represent the relationships between month names and day names with sufficient stability to permit the manipulations involved in running the blend. ${ }^{4}$

Japanese youngsters use this technique to respond to exam questions. The examinations are intended to assess the intellectual abilities of the students. The students appear to have performed complex reasoning, when in fact they have simply done some clever situated seeing. I believe that this general phenomenon is so widespread that much of human intelligence relies on 'tricks' of this kind. To make that case, let me introduce some more examples.

\subsection{Micronesian navigation}

Micronesian navigators routinely sail hundreds of miles out of sight of land without the use of charts, tables, or any of the other instruments deemed necessary by Western navigators (Gladwin, 1970; Lewis, 1976; Sarfert, 1911). Instead, they use an elegant system of superimposed mental images (Hutchins, 1983; Hutchins and Hinton, 1984; Hutchins, 1995). The rising and setting points of selected stars anchor these mental images.

The techniques used by Micronesian Navigators are quite complex. It takes many years of study to master the knowledge and skills required to sail with these techniques. Here I can only briefly sketch a technique that is used to express the position of the canoe during a voyage.

For every voyage between two islands, there is another island off to the side of the course, called the etak island, that the navigator keeps in mind. Since these navigators do not have charts, they do not think of the voyage in terms of the motion of the canoe between the origin and destination as is so natural for those educated in the Western tradition. Rather they imagine the voyage in terms of the movement of the etak island. They know the star bearings between all pairs of islands in their vicinity. This allows them to hold course while sailing to their destination, but it also allows them to imagine that at the beginning of the voyage, the etak island will be at the star bearing of the etak island from the origin. At the end of the voyage, when the canoe has reached the destination, the etak island will be at the bearing of the etak island from the destination. Thus, during the voyage, the etak island appears in the navigator's imagination to move back along the horizon. In fact,

\footnotetext{
${ }^{4}$ With practice, one could conceivably do this manipulation on an imagined hand. The need for practice highlights the need for stability in the representation and shows why having the hand in the perceptual field where it can serve as a material anchor facilitates the process.
} 


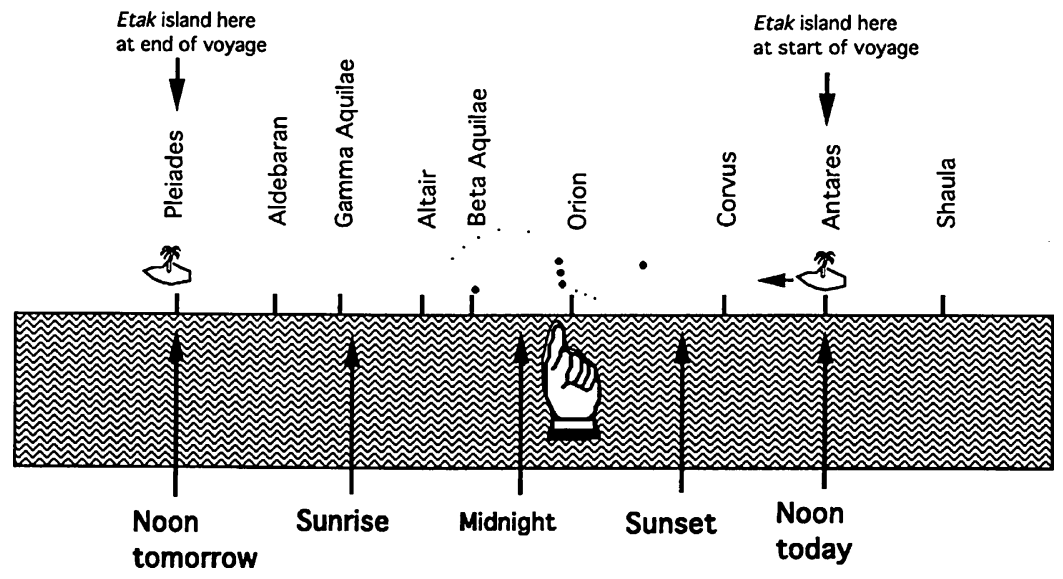

Fig. 6. Just before midnight, the navigator points to the etak island. All he needs to do is point to the location of the current time on the time scale that is superimposed on the spatial landmarks provided by the star points (from Hutchins, 1995).

Micronesian navigators say that once out of sight of land they imagine the canoe stationary under the stars while the islands move. The etak island is under one star at the beginning of the voyage and under another at the end of the voyage. By superimposing the imagined movement of the etak island on the frame of the star bearings, the Micronesian navigator creates a model of the voyage that he can see and manipulate from his point of view on the deck of the canoe. The navigator also knows how long to expect the voyage to take, so he can superimpose temporal landmarks on this image of the movement of the etak island as a model of the voyage. As shown in Fig. 6, this produces a set of correspondences between the location of the canoe (as expressed by the location of the etak island) and time. Using this image, the navigator can accurately point to the etak island at any time during the voyage.

The input spaces include the star points defined by the rising and setting of stars (this structure is the material anchor), the movement of the etak island as embodied in the fictive motion of the etak island trajector along the horizon, and the temporal landmarks. By blending all of these into a single image, the navigator constructs an analog computer.

This system involves the opportunistic use of material structure as an anchor for the superposition of structure from several input spaces. The model is 'run' by imagining the superposition of several kinds of structure thereby producing a blended space in which the ability to infer location from time is an emergent property. The use of this very complex blended space depends on the stability of the representations of precise spatial relations, which would be impossible without the material anchor provided by the star points. Unlike the case of the Japanese hand calendar, the opportunistically used patterns of star points also carries part of the content of the task domain.

This example is especially useful in illustrating the power of conceptual systems to organize thought. Western researchers have questioned the rationality of the Micronesian navigators, saying that their techniques are logically incongruous, or that the Micronesian navigator "does not let logical consistency or inconsistency, insofar as he is aware of them, 
interfere with practical utility" (Gladwin, 1970: 198). However, it can be shown that the models used by Micronesians operate in a different frame of reference and on the basis of different fundamental representational assumptions from those that are common in the Western world (Hutchins and Hinton, 1984). Many inferences that are trivial in the Western way of thinking are nearly impossible to make in the Micronesian system. This accounts for the Western researchers' doubts about the logical abilities of the Micronesians. Conversely, however, the inferences that the Micronesian navigator effortlessly makes in his frame of reference about the location of his vessel and the surrounding islands are nearly invisible to Western researchers. The ability to make inferences in the two systems depends on the culturally conventional conceptual models in which the problem is framed. The organization of those models relies on both the organization of conceptual content and on the structural opportunities presented by the material world.

The two examples given above involve mental models that are anchored to physical structures that exist in nature independent of the cultural activities that have appropriated them. Sometimes, of course, cultural models are embodied in the physical structure of created artifacts.

\subsection{Medieval tide computer}

Frake (1985) describes a medieval tide computer.

The wind rose is an ancient schema that, for most of its history and in most places had nothing in particular to do with representing knowledge of the tides. It was used, first and foremost as a way to express direction. Secondarily, it was used to express solar time ... In whatever manner time was determined at some moment, it was thought of and expressed as a compass bearing. The sun bears south at noon. It was therefore thought of as being north at midnight, east at 6 a.m. and west at 6 p.m. Only the first of these bearings is of any use in northern Europe for determining time. The other bearings were ways of expressing time. (Frake, 1985: 264)

Fig. 7 shows the superimposition of two kinds of structure: the temporal structure of the 24-h solar day is superimposed on the 32-point compass rose. This yields a set of correspondences between direction and time. By blending the conceptual structure of time with the material structure of the compass rose, the navigator can experience direction as an expression of time.

Just as the bearing of the sun is an expression of solar time, the bearing of the moon can likewise be seen as an expression of lunar time. The tides result from the gravitational pulls of both the moon and sun, but the effects of the moon dominate. Although the tide does not simply follow the moon in any obvious manner, the phase of the tide at any particular place is always the same when the moon is at any given bearing. That is, for any particular location, the high tide always comes at a particular lunar time. Medieval mariners referred to the lunar time of the high tide at a particular place as the "establishment of the port." Armed with this knowledge and knowing the phase of the moon, a navigator can easily compute the solar time of high tide in the port.

For example, consider the case where a navigator knows that the tide will be high in a given port when the moon bears WSW. If the moon is new, then solar time and lunar time 


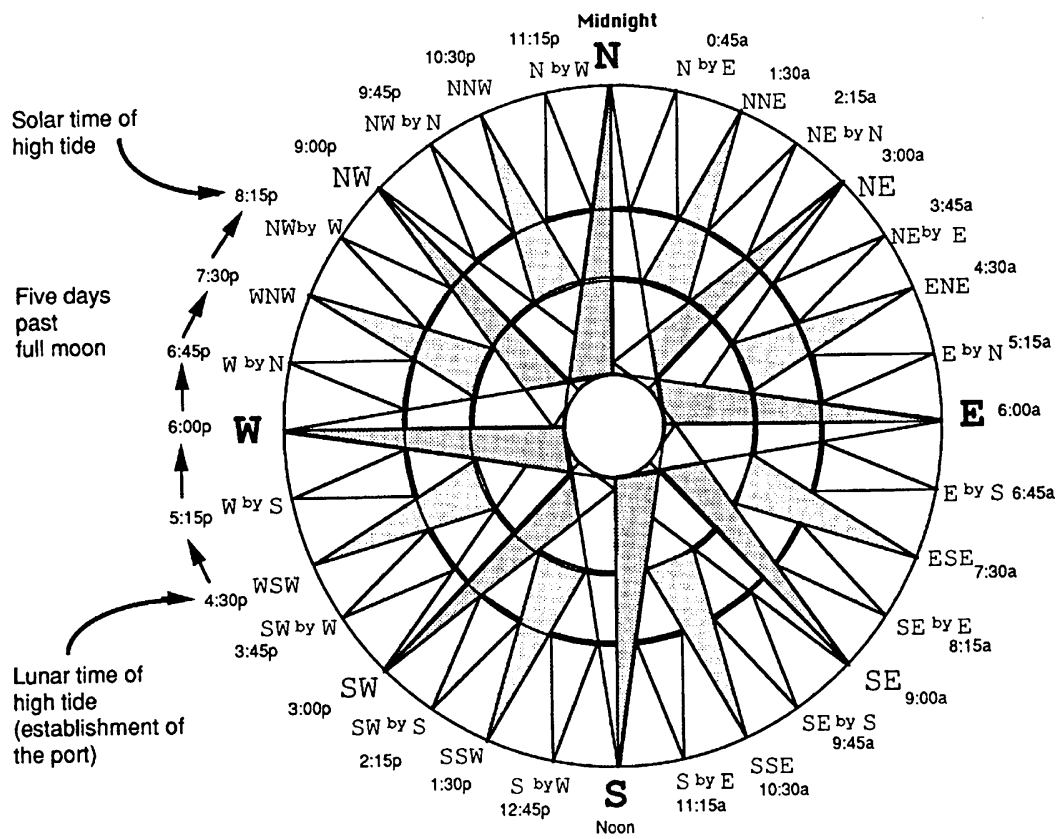

Fig. 7. Computing the tide five days after the new moon for a port with an establishment of WSW.

are the same, and high tide will occur when the sun bears WSW, or 4:30 p.m. If the phase of the moon is other than new or full, the navigator will have to determine the relation of solar time to lunar time. Dividing the 24-h day into 32 equal parts yields intervals of 45 min each. Each day, the moon, and the tide following it, lags behind the sun by about $48 \mathrm{~min}$. The sailor can compute the approximate solar time of high tide by counting one point of the compass for each day after the full or new moon. This computation is the elaboration of the blend.

It is the relationship between determining direction and determining time that makes the use of a single schema, the compass rose, appropriate for representing both direction and time. But the compass rose is not a time-finding instrument. It is a very abstract model, a cognitive schema, of the relations of direction and time, of solar time to lunar time, and of time to tide. It is an etak of medieval navigation. (Frake, 1985: 266)

The use of the medieval compass rose in the prediction of tides is a fine example of the empirical construction of an artifact in the absence of a theory of the phenomenon it permits navigators to predict. In this case, the compass card provides a stable spatial structure onto which conventional techniques of imagining solar times produce a composite image of direction and solar time. Lunar time, which is closely correlated with the tides, is determined by locating a landmark and counting with respect to that landmark a number of points appropriate for the phase of the moon.

The utility of the correspondence between the amount of solar time represented by two adjacent points of the compass and the change in the relation of lunar to solar time each day 
depends on the serendipitous fact that the compass divides the day into 32 equal segments of $45 \mathrm{~min}$ each and the moon lags 1/29th of a day (or about $48 \mathrm{~min}$ ) behind the sun each day. As was the case with the Japanese hand calculator, the computation is done and the blend is 'run' in this system by locating a convenient landmark (the establishment of the port) and counting days before or after the full or new moon with respect to that landmark. Again, the memory loads can be reduced by first selectively mapping from the input spaces to locate the landmark, then performing the counting operations (following an imagined trajector) on the physical structure, and finally selectively mapping from the input spaces again to identify the relevant properties of the location identified by the counting operation. The compass rose as a material anchor stabilizes the conceptual relations among solar time, lunar time, and tide so that the complex operations involved in running the blend can be performed.

These examples are from somewhat exotic domains. Navigation is different from other human activities in the sense that it is a high-stakes activity and the value of finding and saving reliable regularities in the task environment is greater in this domain than in many others. Other high-stakes activities show similar cultural developments. Of course even these specialized activities are more representative of everyday human cognition than most laboratory experiments are. The special nature of these tasks still raises the question of the extent to which these examples are representative of the role of material anchors for conceptual blends in everyday life. To address this, let us turn to one of the most widely used artifacts of modern life, the clock.

\subsection{Clocks, dials, and sliderule}

There is an obvious link between the medieval tide computation and the clock face. The compass rose with times on it is a full sibling to the sundial and the sundial is a direct ancestor of the clock. Both the compass-rose-mapped-with-time and the sundial are blends of the timeline with the direction frame. One is adapted to the ecology of maritime concepts and the other to the cognitive ecology of landlubbers. Reading a clock is a process of running a very complex nested conceptual blend. It is possible to decompose the clock face all the way back to the gnomon and the number line, but of course, it is not necessary to know this decomposition or the history that resulted in the layered composition of spaces that make up the clock face in order to read the clock.

The strategy of mapping numbers onto a circular line is common in dials and instruments of many kinds (Frake, 1994). This is especially apt when the numbers represent a quantity with periodic expression such as time. These instruments rely on a stable anchor (with dynamic moving parts) onto which images of conventional structure are mapped to compose a complex blend. The movement of an imagined trajector gives meaning to the momentarily stationary watch hands, which "go around" to use a fictive motion description, but the actual movement of the hands is necessary to the operation of the device since we are not able to imagine the velocity of movement of the hands with sufficient accuracy to have that dynamic image do the work of a clock.

As a final example of a conceptual blend with material anchors, consider the sliderule. In a sliderule the abstract quantity, logarithm of number, is mapped onto an extent of space. Two such spaces lying next to each other make it easy to add together the extents of space 
corresponding to the logarithms of two numbers. Since the addition of logarithms is an implementation of multiplication, the extent of space corresponding to the sum of the extents of space of the logarithms of the two numbers corresponds to the logarithm of the product of the numbers. The input spaces in this blend are the two representations of the logarithms of the numbers to be multiplied. The blended space is composed by positioning these two spaces with respect to each other in such a way that the total extent of the sum of the extents of space can be seen. The blend is run by reading the product as a number on the scale at the appropriate place. In this case, a specially designed device is manipulated to form the conceptual blend because it is impossible to imagine these conceptual relations with sufficient stability and precision to run the blend using mental resources alone.

\section{Discussion}

Fauconnier and Turner (2002) devote a chapter to "things" in which they draw upon my work on material anchors. They discuss the clock face, other gauges and the method of loci along the lines I have presented here. They also extend the analysis to graves, headstones, dead people, money, and spoken and written language. They justify the extension of the material anchors framework to these phenomena by assuming that material anchors are "objects that prompt for elaborate conceptual integration networks." This broad definition seems to imply that every symbol and sign is a material anchor for a conceptual blend. Up to this point I have tried to show that for many materially-anchored blends, the cross-space mappings are not representational. Let us now take up cases where material structure represents conceptual structure.

\subsection{Material anchors that represent conceptual structure}

Is spoken language a material anchor? Fauconnier and Turner seem to think so, but this claim requires reflection, and a simple yes/no answer misses much of interest here. The minimal informative structure in a material space is individuation, the notion that some material element is different from or distinguishable from other elements. Taking a word (as a material form, either spoken or written) by itself as a material anchor involves this sort of simple mapping. The conceptual space is the lexical contrast set for the word, the other words that might have occurred, but did not. This conceptual space is mapped into the material anchor of the word as a pattern of sound or of written shape. For symbols that have arbitrary relations to the things they denote, the material structure provides only the perceptual identity of the physical form as distinct from other physical forms. Thus, a word

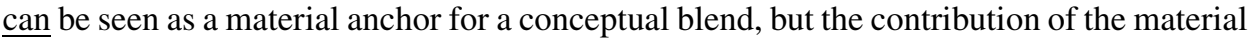
medium to the blended space is minimal. In the framework developed here abstract symbols that have arbitrary relations to their referents will appear as the weakest type of material anchor.

Linguistic units larger than the word may make use of more interesting material structure. Grammatical forms, such as agreements between modifiers and words modified, between predicates and subjects, and between functions and arguments, may rely on a greater contribution of the material form. For example, when two clauses in a sentence 
describe events, the default mapping is that the event described by the first clause happened before the event described by the second clause (Taub, 2001: 25-26). The conceptual relation of modifier to modified, for example, is mapped into the physical structure of word order in many languages. Of course, this conceptual relation may also be supported by other structure such as lexical or morphological cues.

Thus, it may make more sense to say that the temporal or spatial organization of a spoken or written sentence can be a material anchor for some portion of the grammatical conceptual structure, than it does to say that a word is a material anchor for the concept it represents. This is because in the case of sentence structure, more complex aspects of the material organization are involved. No doubt the fact that spoken language has material form was important in the development of language, as the material forms provided anchors for concepts and conceptual relations.

\subsection{Material anchors are not merely signs}

In a recent note, Brandt (n.d.) discussed the notion of the compass as a material anchor. Brandt emphasized the role of the compass needle as a probe that brings information from the "hidden side of reality" to "our side of reality". Focussing on the compass needle and jumping to the interpretation of the compass system as a probe presupposes, and thereby overlooks, the process that I had hoped to highlight with the phrase, "material anchor." From the point of view of material anchors, the compass rose, and not the compass needle, is where the action is. The complex set of spatial relations among the directions (however the frame is conceptualized, e.g., points of the compass, degrees, etc.) would require a huge investment of resources to maintain and operate upon as a purely mental image. In order to produce and manipulate a stable representation of the conceptual elements involved in reasoning about directions, the elements must be somehow held (or anchored) in place. This holding-in-place is accomplished by mapping the conceptual elements onto a relatively stable material structure, the compass rose. Even when an experienced navigator imagines the compass rose, it is a schematic image that has been simplified in ways that make it easier to imagine stably.

The focus on the compass needle rather than the compass rose lead Brandt to assert that "'Material anchors' are signs, and their meanings may all be understood by similar analyses" (Brandt, n.d.). While this does seem to be consistent with the interpretation that Fauconnier and Turner have given the phrase 'material anchors', I do not use it this way. Because a sign is a pattern in a material medium that captures a minimal aspect of the conceptual domain, a sign is a very weak form of material anchor.

\subsection{A continuum of anchoring forms}

Conceptual models embody/express/hold constraints among conceptual elements. In order to play a role in a reasoning process, a conceptual model must be cognitively stable. That is, it must maintain its system of constraints while being subjected to mental (or physical) manipulation. The stability of some conceptual models is provided by their simplicity. Very simple trajectories of attention are easy to remember and apply. The stability of more complex conceptual models is sometimes provided by their being 
embedded in conventional (culturally shared) well-learned and automatically applied internal mental structure. A conceptual model with these properties is a cultural model. With enough practice, support, and motivation, even complex and nearly arbitrary mappings can be learned. In a system of cultural models, each model constrains and is constrained by a rich network of relations to other models in the cultural belief system. It is possible to reason efficiently about complex problems when they are expressed in terms of familiar cultural models. Part of the cognitive power of metaphor derives from the fact that it is possible to reason effectively about unfamiliar concepts, which would otherwise be unstable, if they can first be blended with stable familiar concepts.

Other conceptual models achieve stability by virtue of being blended with an external physical medium. Problems that are too complex to hold in mind as a cultural model, and possibly some that are too complex to express at all in internal conceptual models, can be expressed and manipulated in material structure.

The examples presented in this article illustrate a continuum of cases from those in which external media provide little or no structure of the model to those in which virtually all aspects of the conceptual model are embodied in the structure of external media and the cognitive operations performed on the model are implemented as physical manipulation of material structure. The principal cognitive effect of using conceptual models is achieved by building the constraints of the task into the structure of the model. This is true whether the conceptual representation is entirely a mental image or if it is embodied somehow in physical form.

In the first examples, method of loci, intelligent use of space, and the Japanese hand calendar, the contribution of the material medium is only a set of stable spatial landmarks onto which image structure can be attached and held. Next comes Micronesian navigation, in which the physical structure of the rising and setting points of stars provides a set of landmarks that are themselves task relevant content. The environmental structure used by the Micronesian navigators is not designed for the task. It exists independently of this activity, but it becomes 'artifactual' by virtue of the conceptual relationships that the navigators establish between it and other task elements when they project conceptual structure onto it. The star points are opportunistically used natural structure. The compass rose, as exploited by medieval seafarers to reckon the tides is opportunistically used artifactual structure. It was created by seafarers, but not originally created for this use. Finally, there are cases where the structure is designed for particular problems, the sliderule for example.

Along this continuum, as the nature of the structure of the conceptual models changes from strictly mental representations to more external structure, the kinds of cognitive processes that are involved in 'running the blend' also change. In simple cases, it may be possible to perform a cognitive task, say an inference, with these representations simply by imagining the superimposition of elements of structure. In more complex cases, the fact that some of the task relevant structure is crystallized in a material artifact may reduce the demands on memory. Computing on complex mental images that have material anchors permits people to substitute robust and fast perceptual processes for slow and vulnerable conceptual processes. Since conceptual models work by embodying constraints among conceptual elements, both memory and processing loads can be reduced if the constraints of the task can be built into the physical structure of a material device. In this framework 
the design of cognitive artifacts can be seen as shaping material structure so that it accepts the projection of task relevant conceptual structure.

\subsection{Imaginary material anchors}

In some cases it is possible to do the cognitive work by imagining the manipulation of a physical structure. In others, the case of the sliderule for example, it is necessary to manipulate the physical device itself because it is not possible to imagine it accurately enough to be of use. ${ }^{5}$ The cultural process of crystallizing conceptual models in material structure and saving those up through time puts modern humans in a world where thinking depends in significant measure on the availability of a set of physical structures that can be manipulated in this way.

A final turn on this path is that when a material structure becomes very familiar, it may be possible to imagine the material structure when it is not present in the environment. It is even possible to imagine systematic transformations applied to such a representation. This happened historically with the development of mathematical and logical symbol systems in our own cultural tradition. Beginning as external representations physically embodied and operated upon with manual skills, we learn to imagine them and to operate on the imagined structures (Rumelhart et al., 1986). Unfortunately, much of cognitive science is based on the mistaken view that this relatively recent cultural invention is the fundamental architecture of cognition (Hutchins, 1995). The very idea of rationality, as held by gametheorists, economists, and political scientists, is a cultural construct that owes its existence to the ability to create a certain class of materially anchored conceptual blends. It is a mistake to assume that thinking is, in general, a symbol manipulation process.

Which came first, the purely internal models or the materially anchored models? The blending of a simple trajector with a static object (as is seen in fictive motion) is an extremely old cognitive strategy. Wynn's (1989) analysis of stone tools suggests that toolmakers 300,000 years ago were capable of projecting a simple imagined trajector onto the edge of a tool to control a series of blows. Might this materially anchored activity have been a precursor to the ability to form simple internal blends? It is clearly the case today that people are capable of conceptual feats supported by material anchors that could not be undertaken using mental resources alone. This must also have been true in the past. Recently, speculations on the origins of human intelligence have given more emphasis to the demands of social reasoning than to tool making (see Byrne and Whiten, 1988). Perhaps viewing conceptual models in this way will revive the importance of the toolmaking heritage. In any case, it seems likely that patterns of interaction with emergent regularities in the material and social environment provide the basis for whatever truly internal conceptual skills we have.

\footnotetext{
${ }^{5}$ It is easy for an experienced user of the sliderule to represent the $\mathrm{C}$ and $\mathrm{D}$ scales on the sliderule as two adjacent logarithmic scales. I can "see" them that way, and I can even imagine them that way. Conceptually, I also know that the distance separating successive digit labels is less as one moves from left to right on the scale. The distance between 1 and 2 is greater than the distance between 2 and 3, which is greater than the distance between 3 and 4, and so on. Ah, but how much less? To sustain the performance of the computation on the sliderule those metric relationships must be exact. That is why the sliderule scales are printed with tiny tick marks and there is a sliding vertical hairline that preserves spatial relations at the limits of human visual perception.
} 
It is also important to stress the systematic nature of the models presented in this article. This is not simply a set of isolated examples of clever cognitive strategies. Rather, each of the models presented here is a part of an ecology of conceptual blends that have accumulated and been layered, one over the other, to produce complex emergent properties. Of course, it is not necessary for the users of these tools to know the history of the tool any more than it is necessary for us to know the history of the blends that we find in language.

Each of the examples presented here is part of a larger cognitive ecology and a system of cultural models. The examples are also unified by the fact that each illustrates the operation of the cognitive processes known as conceptual blending. Conceptual blending has previously been seen as an entirely internal cognitive process. I have tried to show in this article that the same kinds of processes operate in situations where one or more of the input spaces to the blend contain material structure. This unification is important because it provides an antidote to the false dichotomy between the study of conceptual models and the study of material resources for thinking.

\section{References}

Brandt, Per Age, n.d. On two material anchors. Unpublished email, May 2002.

Brooks, Rodney, 1991. Intelligence without representation. Artificial Intelligence 47 (1-3), 139-159.

Byrne, Richard W., Whiten, Andrew (Eds.), 1988. Machiavellian Intelligence: Social Expertise and the Evolution of Intellect in Monkeys, Apes, and Humans. Oxford University Press, New York.

D’Andrade, Roy, 1989. Culturally based reasoning. In: Gellatly, A., Rogers, D., Sloboda, J. (Eds.), Cognition in Social Worlds. McGraw-Hill, New York.

Fauconnier, Gilles, 1997. Mappings in Thought and Language. Cambridge University Press, Cambridge.

Fauconnier, Gilles, Turner, Mark, 2002. The Way We Think. Basic Books, New York.

Frake, Charles, 1985. Cognitive maps of time and tide among medieval seafarers. Man 20, 254-270.

Frake, Charles, 1994. Dials: a study in the physical representation of cognitive systems. In: Renfrew, Colin, Zubrow, Ezra (Eds.), The Ancient Mind: Elements of Cognitive Archaeology. Cambridge University Press, Cambridge, pp. 119-132.

Gladwin, Thomas, 1970. East is a Big Bird. Harvard University Press, Harvard, MA.

Harwood, Frances, 1976. Myth, memory, and the oral tradition: cicero in the Trobriands. American Anthropologist 78 (4), 783-796.

Hutchins, Edwin, 1983. Understanding Micronesian navigation. In: Gentner, D., Stevens, A. (Eds.), Mental Models. Lawrence Erlbaum Associates, Hillsdale, NJ, pp. 191-225.

Hutchins, Edwin, 1987. Myth and experience in the Trobriand Islands. In: Holland, D., Quinn, N. (Eds.), Cultural Models in Language and Thought. Cambridge University Press, Cambridge, England, pp. 269-289.

Hutchins, Edwin, 1995. Cognition in the Wild. MIT Press, Cambridge, MA.

Hutchins, Edwin, Hinton, Geoffrey, 1984. Why the islands move. Perception 13, 629-632.

Kirsh, David, 1995. The intelligent use of space. Artificial Intelligence 73 (1-2), 31-68.

Langacker, Ronald, 1987. Foundations of Cognitive Grammar, vol. 1. Stanford University Press, Stanford, CA.

Lewis, David, 1976. A return voyage between Puluwat and Saipan using Micronesian navigational techniques. In: Finney, B. (Ed.), Pacific Navigation and Voyaging. Polynesian Society, Wellington, New Zealand, pp. 15-27.

Liddel, Scott, 2003. Grammar, Gesture, and Meaning in American Sign Language. Cambridge University Press, Cambridge.

Nakahara, Hiroyuki, 1996. Japanese Hand Calculator. Unpublished manuscript, University of Tokyo.

Sarfert, E., 1911. Zur Kenntnis der Schiffahrtskunde der Karoliner. Korrespondenzblatt der Deutschen Gesellschaft fur Anthropologie, Ethnologie und Urgeschichte 42, 131-136.

Talmy, Leonard, 1995. Fictive motion in language and perception. In: Bloom, P., Peterson, M., Nadel, L., Garrett, M. (Eds.), Language and Space. MIT Press, Cambridge, MA, pp. 307-384. 
Taub, Sarah, 2001. Language from the Body, Iconicity and Metaphor in American Sign Language. Cambridge University Press, Cambridge.

Wason, Peter Cathcart, 1966. Reasoning. In: Foss, B. (Ed.), New Horizons in Psychology, vol. 1. Penguin Books, Baltimore, pp. 135-151.

Wynn, Thomas, 1989. The Evolution of Spatial Competence. University of Illinois Press, Chicago.

Edwin Hutchins is Professor of Cognitive Science at the University of California, San Diego. He earned a PhD in Cognitive Anthropology from the University of California, San Diego in 1978. In 1985, he was awarded a prestigious John D. and Catherine T. MacArthur Foundation Fellowship for his work on the conceptual structures underlying Melanesian land litigation and Micronesian navigation. Professor Hutchins is known for his research on cognition in real-world settings. His study of the cognitive processes involved in ship navigation resulted in the publication of the book, Cognition in the Wild. 